\title{
Utopia literaria. Le dépaysement de Jean-Christophe Bailly
}

\author{
Amélie CALDERONE, Université Lumière Lyon 2, France \\ \& Carolina CARNIER, Université Lumière Lyon 2, France
}

Avec la participation de Blaise Djomaleu KAMADEU, Université Jean Moulin Lyon 3

L'identité, il faudrait en premier lieu la relier à ce qui est son véritable terrain, je veux dire l'ensemble (bariolé !) de toutes les possibilités du devenir soi-même.

Jean-Christophe Bailly, Le dépaysement (475)

Véritable questionnement, Le dépaysement est une quête de l'essence de ce que l'on appelle par habitude et/ou par facilité «France », et une enquête. Jean-Christophe Bailly se fait voyageur - Wanderer - et, à travers les lieux, les monuments, la population, ou encore les objets, l'on voit se dérouler sous nos yeux une « histoire des traces » (14) : la reconstitution d'une identité grâce à des indices perçus au fil de voyages menés sur le territoire. L'œuvre se constitue pour une large part comme une enquête, bien que non systématique : elle est essai de « déchiffrement» des paysages français, tentative d'y apercevoir des « échos soulevés en passant » (Bailly, Phrase, 30) à partir desquels « l'intuition d'un récit » pourra naître. Une dynamique spécifique travaille ainsi en profondeur un texte au sein duquel deux systèmes sémiotiques s'imbriquent : le paysage d'une part, diffusant des signaux qui composent une image fluctuante et mobile de la France, image organisée, pour reprendre les termes de Bailly, comme une «pelote complexe et enchevêtrée où époques, affects et dimensions s'entremêlent » (28); l'écriture d'autre part, qui essaye de rendre compte de la multiplicité et de la complexité de ce phénomène.

Mais une continuité entre le monde, la réalité (ces lieux de la France), et le concept (celui d'identité) - autrement dit entre des totalités non interrompues et la langue dans son caractère linéaire, séquentiel, structuré par la grammaire, est-elle possible ? Le langage, grâce auquel des révélations, des caractérisations de ce qui est nommé «France » se font jour, devient, de fait, un «défi »: un «tatouage mobile » (Bailly, Dépaysement, 29) qui doit être à même de donner à voir la nature composite et perpétuellement métamorphique de son objet. À l'instar de Rodin (96), pour qui les cathédrales - bien que disséminées en îlots sur le territoire français - étaient le signe évident d'une liaison ininterrompue entre celles-ci, le pays et l'expression, l'auteur noue au fil de son ouvrage un lien profond entre la langue et l'objet qu'elle entend définir. Il arrime la forme littéraire aux formes appréhendées; créant en 
somme un lien aussi fragile qu'indissoluble entre la littérature et le monde. Certes, la réflexion sur les capacités (ou l'incapacité) du langage à dire le réel est aussi ancienne que commune à bien des écrivains, mais si pour nombre d'entre eux l'expression est un moyen de circonscrire et d'ordonner le monde, le rapport s'inverse chez Jean-Christophe Bailly, lequel organise son écriture en fonction du régime de signes que le paysage, l'architecture et les lieux renvoient. La langue et la forme littéraire qui en résultent, «involontaire refondation rhétorique » (14) est aussi littérairement ambitieuse que risquée.

Si le paysage est langage ${ }^{1}$, la structure textuelle forgée par Bailly tente d'incarner ce mouvement de «glissement » (Phrase, 29) constant, actualisant pourtant l'image multiple de la France qui se constitue dans le voyage. Une relation s'établit subrepticement entre la chaîne des signes perçus qui nous entourent, la notion d'identité qui donne matière à réflexion et qui s'élabore au fil du texte, et le genre littéraire qui conviendrait. Si lire et comprendre un texte ne nécessite pas d'être asservi à ce compulsif besoin de classification générique sur lequel se fonde l'enseignement littéraire français, force est de constater que ces «Voyages en France » ne peuvent que dérouter (au sens étymologique) un lecteur habitué à des grilles de lecture formelles. Nombre de librairies d'ailleurs, organisées selon cette typologie classificatoire, se sont vues contraintes de catégoriser cette œuvre composite parmi les « récit de voyage ». Alors même que Bailly a délibérément refusé l'appellatif « roman ».

Ce simple constat invite à interroger les moyens de comprendre et de caractériserterme cher à l'auteur - ce texte. Comment en effet le situer par rapport à des traditions littéraires ? Et par rapport au projet qu'il se propose, et à la matière qu'il se donne ? Nous souhaiterions montrer qu'avec Le dépaysement, Bailly a l'ambition de fonder un nouveau genre, dans une volonté pour beaucoup redevable à l'influence du romantisme allemand, notamment du modèle novalisien du « roman colossal » dont cet auteur se réclame. Mais s'il est tout imprégné des Romantiques, Bailly pétrit sa forme nouvelle : dans le mouvement d'élaboration d'une utopie littéraire, il convoque également bien d'autres modèles, pour donner lieu finalement à une solution toute personnelle. La question formelle de la caractérisation du genre de cet ouvrage polymorphe qui se pose le plus immédiatement, entraîne la question énonciative de la voix auctoriale, ou plutôt des voix multiples qui se prononcent; à travers elles, c'est une redéfinition du statut de l'écrivain qui nous semble engagée. 


\section{Le dépaysement : un « roman colossal »?}

Face à ce texte étrange et déroutant, le lecteur se voit réduit à opérer exactement comme l'auteur le fait avec son objet : contraint à une attention et à une écoute profonde du texte, il devient de fait attentif aux moindres indices disséminés, susceptibles de l'aider à mieux cerner l'identité du Dépaysement. Or, le modèle dont Bailly se réclame le plus explicitement, c'est celui du « roman colossal » de Novalis :

Face aux éclaboussures et aux caramboles de la cascade [...] j'ai l'intuition, je pense, je vois, j'ai l'intuition d'un récit que je ne pourrai pas rejoindre mais qu'il faudra du moins effleurer - un récit ou, plutôt qu'un récit, un extrait de ce que Novalis appelait le «roman colossal », expression qui depuis que je l'ai découverte [...] s'est toujours portée au-devant des choses aussitôt que cellesci se mettaient à ricocher de plus en plus vite, formant des sortes de fondus enchaînés ou de glissages emportés par un effet dominos qui balayait les hiérarchies et rangements. (Bailly, Dépaysement, 26-27)

Rappelons qu'aux yeux de notre auteur, qui a présenté par ailleurs une anthologie des Romantiques allemands - La légende dispersée -, Novalis est «la conscience même du sens encyclopédique » (20) : ce modèle semble le plus capable de faire lien entre le monde comme totalité et la syntaxe de l'expression. Pour l'auteur, le «sens encyclopédique du romantisme allemand dépasse et oublie celui du XVIII ${ }^{\mathrm{e}}$ siècle », notamment parce qu'au rayonnement de la raison, il substitue « un sentiment, celui d'une constance, celui de la présence d'un indéchiffrable qui li[e] tout» (21), faisant de fait «porter l'accent sur les conducteurs, la vitesse, sur les mouvements qui animent la nature en profondeur » (18). Le « roman colossal » ${ }^{2}$ tel que Bailly l'envisage et semble en rêver, réside «moins dans une immensité massive que dans la sensation, infiniment palpable, d'une immensité répartie et présente à même la séquence $»^{3}$ (Dépaysement, 27). En d'autres termes, le modèle invoqué par l'auteur du Dépaysement est à ses yeux le seul capable d'exprimer l'infini par et dans du fini. Dès lors, l'encyclopédisme sera apte à préserver « du double mal qui pèse sur l'horizon de la pensée : «d'un côté la spécialisation [...], et de l'autre l'ignorance [...].» (Bailly, Légende, 21).

Profondément marqué par sa découverte des Romantiques allemands, Bailly remet en jeu une conception métaphysique panthéiste de l'univers - le monde comme un tout - et les moyens formels de sa transcription littéraire. La référence à Novalis, en particulier, jalonne sa pensée :

C'est le « roman colossal » dont parla Novalis. Il se déploie tout seul, puis des noms le conduisent et, parmi ces noms, les noms de lieux sont les guides les 
plus avertis. Ils servent de relais et d'appuis, à travers eux on rêve que tout le langage puisse avoir ce pouvoir de localisation extrême et d'enclenchement de la résonance qui les fait ricocher à la surface de la langue et qui transforme chaque lieu-dit en un petit ou en un immense tremplin. (Bailly, Phrase, 68)

L'assimilation et la compréhension personnelle de l'esprit et de la forme novalisiens expliquent l'attention portée à l'Histoire : le lieu c'est aussi du temps. Le dépaysement se présente ainsi comme une succession de fragments de l'Histoire - et/ou des histoires - qui imprègnent les paysages. Si pour Bailly l'identité est une infinité de départs possibles, si pour lui l'histoire est une infinité de latences et le paysage un système infini de signes et de combinatoires possibles, si enfin sous sa plume une «écriture glissée » (Bailly, Phrase, 8) permet d'interpréter cette multitude de matériaux poétiques, c'est bien au sein du « roman colossal » que les signes communiquent selon ces liens mystérieux et analogies secrètes qu'il s'attache à révéler. Le dépaysement s'érige sur cette myriade de signaux qui s'entrecroisent et composent une trame, qui raconte et donne à voir une autre image, fût-elle évanescente, de «la France ».

Le « dépaysement » littéraire que propose l'œuvre de Bailly, est ainsi une écriture du déplacement, des «glissements » inattendus d'un lieu vers un autre, ou des infiltrations à partir du lieu où l'on se trouve, provoquant des « départ[s] imprévu[s]» (29) de l'écriture. La quête de l'existence d'une identité devient un parcours de résurgences au travers d'une écriture elle-aussi «glissée », «coulée », véritable ligne fuite en vertu de laquelle le passé devient une présence résonante, une «suite sans fin » de «dormance[s] » (151) du « roman colossal » toujours en puissance. L'Histoire, les histoires ou encore les éléments constitutifs d'une légende sous le signe de la «partance » sont retracés à travers des vestiges porteurs de mémoire imprégnée dans les lieux. Chaque objet considéré devient une pelote dont il suffit de tirer un fil pour amener à soi les infinis replis du temps et les multiples souvenirs, images et sensations qu'il charrie. Le passé reste ouvert et s'exprime - ou effleure à la surface du présent - par le biais d'infimes et ténues réminiscences, selon un « régime de traces dont certaines sont encore à venir »(Bailly, Dépaysement, 122). Bailly nous donne à voir la force émanant du paysage jusque depuis ses aspects les plus dérisoires ou triviaux, la «vie » qui flotte sur les vestiges et qui demeure malgré le passage du temps et de ses métamorphoses (147). De ce point de vue, le « roman colossal» de Bailly est aussi, sinon d'abord, celui qui est généré par les «petits riens » de la vie quotidienne :

[...] une cour ou un jardin dont on ne saura rien d'autre que l'effleurement mais qui, par une tonnelle, une table et quelques chaises, ou moins encore, 
donne consistance à une rêverie qui ne dure pas mais qui s'anime comme un infime départ vers une autre existence - petite fiction sans personnages et sans cause dont on est le narrateur passager et qui est l'un des plus grands charmes de la province $[\ldots]$. (252)

Les indices donnés par les lieux deviennent sous la plume de l'auteur chant mystérieux, émané de ces «choses» sublimées par Ponge, et sont de fait susceptibles d'occasionner un départ inattendu «vers une autre existence », comme l'entendait Novalis, pour qui chaque objet ou chaque être est porteur de cette virtualité. Les signes qui nous entourent, dans une double dynamique de dispersion et de communication constante, se détachent de leur ensemble originel pour agir au sein du paysage et le transfigurer. Ce sont dès lors de nouvelles relations de sens que met au jour le «roman colossal », dans l'esprit également des «correspondances» baudelairiennes. La fiction ainsi (re)composée, le discours établi à partir de ces traces communicantes, se fera révélation, véritable moment d'épiphanie capable de métamorphoser le regard usé que le lecteur aurait spontanément porté sur un objet. Le dépaysement collectionne ainsi ces frêles et intenses moments épiphaniques qui avaient fait aussi l'enjeu du travail de James Joyce. Mais à l'ambition de représentation totalisatrice du lieu (France) à travers les lieux, Bailly, adaptant le modèle novalisien, accumule les «brin[s]» de la «gigantesque meule de foin», et amoncèle une suite d' « infime $[s]$ séquence[s]». D'où, au fondement de sa création, l'idée que « chaque parcelle de réel découpée dans la séquence [devienne] un monde : de telle sorte que l'on n'aura pas ici le roman, mais seulement son schème » (27). Chaque chaîne de signaux qui déclenchera - ou sera susceptible de déclencher - des discours nouveaux et singuliers : autant de fictions en puissance dont l'impossible ensemble serait une partie infime du roman totalisateur de Novalis. Plus donc que le «roman colossal» lui-même, c'est le cheminement vers une possible réalisation de ce modèle littéraire, c'est la quête de ses potentialités et de ses fécondités sonnant comme de formidables promesses qui intéressent notre auteur.

La définition d'une essence française se voit ainsi étroitement liée à un modèle philosophique et littéraire, en vertu d'une écriture toujours en quête de sa forme parce que sans cesse sur le départ ou, plutôt, toujours grosse des départs possibles; une écriture des correspondances secrètes qui sous-tendent un monde en mutation constante, spatialement comme temporellement dispersée, à la façon des signes discrets qui lui donnent vie et voix ${ }^{4}$. Bailly s'approprie moins le «roman colossal» que ses «partances » et ses «dormances ${ }^{5}$ » comme autant de possibles. Euvre délibérément en inachèvement perpétuel - reflétant son 
objet ondoyant et in(com)préhensible -, refusant la clôture et la rigidité d'une forme générique de classification textuelle, Le dépaysement est un ouvrage profondément ouvert à l'altérité littéraire, forgé par l'assimilation d'une multitude de matrices selon un modelage singulier, seul apte, pour l'écrivain, à exprimer (utopiquement) son utopie ${ }^{6}$.

\section{Le dépaysement : une ouvre ouverte}

Si le « roman colossal » poursuit par certains aspects l'objectif encyclopédique initié par les philosophes des Lumières et de la Révolution française, Novalis et les divers groupes du Romantisme allemand se distinguent de la philosophie française des Lumières en ce que leur volonté totalisante « échappe au piège de la forme et de la systématisation et s'efforce de maintenir sans cesse - dans les signes même-le rapport avec le monde frémissant de la nature, avec l'instabilité des passions et des mouvements qui gouvernent l'existence des hommes » (Bailly, Légende, 28). Aussi l'«encyclopédie » demeure-t-elle toujours ouverte sur le monde et les signes qu'il émet, et l'ascendance sous-terraine du romantisme chez Bailly est-elle sensible jusque dans son appréhension - et sa préhension - sensible et intellectuelle du monde. Dans cette perspective, toute forme est susceptible d'être intégrée, ingérée, Le dépaysement se faisant l'écho d'une hantise presque pathologique de la clôture. Si l'ouvrage de Bailly est inclassable, c'est parce qu'il est un appel constant - véritable siphon textuel - aux modèles possibles :

Pour ce qui est du genre, mon désir, s'affinant et se précisant au fur et à mesure que les choses avançaient, aura finalement été celui de parvenir à un livre composite, embrayant diverses vitesses d'écriture, tenant par certains côtés de l'essai et par d'autres du journal de bord, du récit et de l'embardée, voire, épisodiquement, du poème en prose, tout ce qu'on voudra mais en tout cas tendu par une injonction plus brutale - non pas le réalisme bien sûr, plus personne n'y croit, mais le désir que la forme verbale, quel que soit par ailleurs son travail, réponde le plus exactement possible à une dictée extérieure venant des choses rencontrées, le modèle, non verbal, étant ici celui de la photographie et de sa teneur indicielle, le petit écran baladeur des appareils numériques étant compris dans le lot. (14-15)

C'est parce que son ambition est totalisante - intégrer le monde, en l'occurrence dans Le dépaysement les éléments constitutifs de l'identité française, dans le cadre de la phrase, dans des « séquences » (443), autrement dit intégrer la masse continue du réel dans un moule linéaire et nécessairement porteur de fragmentation -, que l'œuvre de Bailly se fait poreuse aux possibles littéraires, en toute conscience. Le texte est ainsi non seulement « composite », mais également méta-littéraire, qu'il s'agisse pour Bailly de refuser des modèles - «[I]1 
n'était de toute façon pas question, dans ce livre, de faire des portraits de villes » (378) - ou d'en invoquer d'autres.

Relevant en partie du genre du récit de voyage, le texte s'en distingue toutefois parce que l'excursion de Bailly est moins un tableau géographique - façon Vidal de la Blache (192) - ou encore touristique - façon Stendhal dans ses Mémoires d'un touriste que notre auteur cite abondamment - qu'un itinéraire de quête : celle d'une identité-France dont on cherche à percevoir et sentir les traces au cours de pérégrinations. Exploration toute singulière, mêlée d'éléments autobiographiques, sous le signe aussi de Frances A. Yates (150) : outre les objectifs fixés en amont de l'écriture, notre voyageur erre, décide inopinément de longer un cours d'eau - alors surgit dans le livre le «temps des rivières » (298) - suit les traces des amitiés ${ }^{7}$ - une galette des rois partagée engendrera le «voyage de la fève » (28), un ancien élève lui aura fait découvrir un modèle d'élevage à Vernand (423) des nécessités professionnelles - à la cité des Gibjoncs de Bourges, devant servir d'appui aux projets d'élèves en école de paysage de Blois (463) - mais également celles de la « rumeur d'enfance » (478) et des souvenirs. Intime et subjectif, l'itinéraire de Jean-Christophe Bailly est en outre médiatisé par l'imprégnation culturelle d'un homme qui a grandi et vécu dans le pays où il voyage - ainsi lorsque le «transparent de Carmontelle » permet une lecture renouvelée des jardins ouvriers de Saint-Etienne (66). L'ouvrage oscille ainsi entre la ligne d'horizon (et de fuite) donnée par des objectifs (de lieux, de visites) qui entraînent en fait une sorte de circonvolution, et un objectif - celui-ci politique : l'identité. Parfois livré au hasard, Le dépaysement se présente comme un vaste «nous verrons bien » (298), et si l'auteur a entamé son projet avec une liste de lieux devant servir de colonne vertébrale à son itinéraire, les schémas de la quête et de l'enquête inhérents à son dessein ont nécessité non seulement des ajustements, mais également une écoute attentive des potentialités de l'errance :

Très divers aura d'ailleurs été le destin des noms de lieux figurant sur cette liste extensible et sans fin raturée: tandis que certains ont effectivement occasionné des voyages (de découverte ou de retour) pour devenir des chapitres de ce livre, d'autres ont été abandonnés en cours de route. Mais, surtout, d'autres, imprévus au départ, sont venus s'imposer, une logique du tuilage - tel chapitre entraînant tel autre - s'étant mise en place dès lors que l'écriture eut vraiment commencé. (13)

Bailly entrecroise alors des regards tout à la fois poétiques, philosophiques, scientifiques et critiques, ou encore contemplatifs, lui qui s'attache à «suivre des lignes errantes et [à] ne craindre ni les sautes, ni les recoupements, ni même les redites » (398). Par 
des traits qui relèvent du carnet de bord ou du journal de voyage, la fiction est livrée au lecteur d'un ouvrage en train de s'écrire sous ses yeux - «je me suis trompé » (76) - d'un texte délibérément maladroit, comme le fut la «marqueterie mal jointe » de Montaigne (Essais, 1008) procédant «à sauts et à gambades» (1040) par un art conscient de la digression: «et voilà que je me retrouve avec une Moselle de conte russe !» (Bailly, Dépaysement, 197). Bailly semble sans cesse conduit par l'ambition de dire exhaustivement le monde, d'où la densité de son texte, lequel contient également des listes homériques ou rabelaisiennes d'objets - par exemples d'animaux (433) - ou cède parfois à la tentation du « tout noter » objectivement, parfois dans un style télégraphique, comme c'est le cas durant le voyage en train entre Arles et Mulhouse (31). Multiple, l'œuvre de Bailly est ainsi une véritable « réaffirmation du caprice et du désordre » (201).

Il n'est donc pas surprenant que l'œuvre tienne également de la «rêverie d'un promeneur solitaire » par son aptitude à pratiquer la rêverie par la promenade et la flânerie : «[...] une émancipation est nécessaire, et [...] une image dépliée de la ville contient les germes d'une 'flânerie élargie', si l'on peut reprendre ici le motif de l'amplification que Novalis souhaita à la poésie » (Bailly, Phrase, 196). La notion de «promenade » en état de rêverie selon Rousseau, mais aussi la notion de «flânerie » plus spécifiquement liée au monde de la ville, que Bailly analyse en détail dans La phrase urbaine, notamment en référence à Baudelaire et Walter Benjamin ou encore au mouvement surréaliste (dont l'auteur fut marqué dans sa jeunesse), sont porteuses d'une disponibilité et d'une force de perspicacité (au sens étymologique) au milieu du monde et de ses signes ; un mouvement d'amplification s’y ajoute selon Novalis, avec la notion de « flânerie élargie ».

Le dépaysement devient donc parfois un véritable roman; non pas roman « romanesque » tendu par une intrigue trépidante et des effets dramatiques, mais roman des romans possibles parce que chaque regard est porteur d'une fiction en puissance, à l'instar de ce plan de Bordeaux qui, rassurant dans son embrassement apparent d'une totalité massive, en réalité « déclenche [...] le roman au lieu de la calmer»(Bailly, Dépaysement, 21) par ses seules références toponymiques. Et ce, jusqu'à parfois réécrire l'Histoire sur le mode du « et si »-1'autre règne, rêvé, de Louis XIV (209) ou le monde d'aujourd'hui si les familistères de Guise s'étaient répandus (371) - voire jusqu'à tenter de combler ses vides : «Et 1'on peut rêver qu'à ce moment un éclair de lucidité ait traversé son esprit [à Napoléon III, en partance 
pour l'île d'Elbe], lui laissant entrevoir tout ce qu'il avait dû perdre pour atteindre cette gloire d'où maintenant il se retirait $[\ldots] »(114)$.

Roman des romans possibles, Le dépaysement l'est aussi parce que, in fine, le lecteur ne saura plus vraiment faire le distinguo entre la réalité et sa contemplation, sa description minutieuse, son analyse, mais aussi sa sublimation : "C'est exactement comme dans un roman - il arrive que des scènes vécues, qui n'ont rien de spectaculaire, se détachent d'ellesmêmes pour produire une sorte de fiction stationnaire qui est aussi comme un plan au cinéma $[\ldots] »(87)$. Le monde devient roman(s) pour celui qui tente d'écrire le roman du monde, et pour le lecteur qui l'accompagne dans ce voyage. Jouant de formes littéraires entre référentialités réelles et cadres imaginaires, Le dépaysement intègre aussi des mouvements poétiques selon le registre mentionné du «poème en prose ». En vertu d'une poésie du quotidien, du trivial et de l'ordinaire, voire d'une prose poétique de la ville qui rappelle le projet de Baudelaire, Jean-Christophe Bailly parvient à habiter non seulement le monde en poète, mais aussi à habiter, dans le lieu même du texte, un monde tramé par la parole des auteurs qui l'on habité auparavant. L'hommage à «Rimbaud parti », par exemple, témoigne de la tentative de percevoir et de retranscrire le «chant cassé » d'un paysage autrefois rimbaldien. L'usage d'une métaphore musicale («chant», « ritournelle ») rappelle alors l'idéal - fragile - du poète oraculaire, voyant et porte-voix, perçu au sein du paysage dans un phénomène de musicalité à peine audible :

Et c'est cette manière de porter le chant, non comme un souvenir et certainement pas non plus comme une promesse, qu'avec les moyens du bord - mots ou images - on cherche à capter : [...] comment il est possible, dans cette espèce de ritournelle enfoncée à même les mares et les ronces des sous-bois, d'apercevoir, peut-être, ce fond de vérité et même de vérisme qui trame l'espace de la voyance et de l'oracle. (135)

Mais si Bailly convoque sur la scène de son livre des modèles que l'imaginaire rattache à la littérature - carnet de notes, journal de bord, récit de voyage, roman, poème en prose, poésie - Le dépaysement relève également de genres philosophiques et politiques, au registre cette fois de l'essai. Réflexion critique sur la polis dont le «fil rouge politique » (277) aboutit à l'hypothèse finale du « bariol $^{8}$ », l'œuvre est liée à l'actualité de son temps celle notamment de la création d'un « Ministère de l'identité nationale »-, datée - « rédigée entre le printemps 2008 et l'automne $2010 »(7)$ - et entend apporter sa contribution à la mise au jour, voire à la définition (finalement avec l' «Hypothèse du bariol ») d'une identité française qui fait débat. Mais au lieu d'un seul « essai » univoque, peut-être alors est-ce le 
modèle des Essais montaigniens qui est sous-jacent (quoique Montaigne ne soit jamais mentionné) : essais pluriels dans leur aspect tournoyant, dense, aussi poétique qu'intellectuel, politique et philosophique, fragmentés dans leur tentative d'embrassement exhaustif, retors, faits de plis et de replis. Montaigne, Bailly élaborent tous deux, en somme, une masse nodale irréductible à un seul modèle. S'il s'agit d'un « essai » que ces «Voyages en France », du moins est-ce plutôt au sens de «tentative » : celle de construire un nouveau genre littéraire, seul capable d'exprimer ce quelque chose de fuyant, mobile et presque indicible que notre auteur cherche pourtant à caractériser et mieux, à définir.

Ne serait-ce pas alors une utopie que ce Dépaysement ? Force est en effet de constater que les écrits de Bailly concernant l'utopie politique prennent un sens métalittéraire, ne serait-ce que parce que cette dernière « glisse [...] sur d'autres genres du penser (la rêverie, la fantaisie $)^{9} »$, et parce qu'il en appelle à «une utopie non captive, et peut-être même un idéal non fixé en un point de la paroi du temps ${ }^{10} »(150-151)$. Si le «bariol » est une création de l'auteur, un mot intégrant le multiple, connotant la bigarrure, lui-même forgé entre deux langues mais aboutissant à une proposition d'unité, Le dépaysement est le résultat de cette même recherche à l'échelle textuelle. Au cours de l'œuvre, une u-topie-au sens étymologique aussi-du mode d'écriture se construit, en vertu d'une quête, sous le composite, d'unité : thématique, politique, littéraire. Aussi Le dépaysement est-il profondément un ouvrage de ce lien si cher aux Romantiques, sans cesse en cours de définition d'une unité - qui ne serait ni uniformité ni unicité -, au sein même de son ouverture. Le texte, selon l'étymologie même du mot, recouvre de fait sa valeur originelle d'une « grammaire générative ${ }^{11}$ » d'un tissu qui se noue grâce à de multiples fils, mettant au jour les ligatures secrètes qui le composent. C'est un des sens à accorder aux nombreux rappels et aux annonces qui jalonnent l'œuvre afin qu'elle parvienne, fût-ce imparfaitement, à s'ériger en un tout ${ }^{12}$.

À l'image du pays appelé de ses vœux par l'auteur, il y aurait donc au fondement de l'écriture du Dépaysement une «rationalité nouvelle qui, fondée sur le multiple et redoutant les figures et les masques de l'Un qui [...] est toujours redoutable, saurait rééquilibrer le national à l'aune des arrivées et des désirs, et non à celle des nostalgies » (Bailly, Dépaysement, 279). Jean-Christophe Bailly, recoupant par là une utopie politique avec une utopie littéraire, parvient à rendre et un pays et une littérature «exotique[s] à [eux]même[s] » (330). S'il dé-payse, s'il fragmente ce que l'on comprend sous un terme un - la 
France - et en déconstruit les archétypes, l'on pourrait surtout dire qu'il «re-payse »: il redonne une signification et une cohérence nouvelles à des termes galvaudés, et apporte une vitalité nouvelle en littérature.

\section{Les voix multiples du Dépaysement : Bailly, ouvrier littéraire polymorphe}

La multiplicité générique du Dépaysement peut se poser à présent dans les termes d'une problématique d'énonciation qui remonte jusqu'à l'auteur en son nom propre, et dans son statut d'écrivain. Il est difficile d'assigner à ce livre une voix auctoriale univoque. Qui nous parle? L'invitation au dépaysement littéraire enjoint à repenser-et ouvrir-le qualificatif d' «écrivain », dès lors qu'on l'applique à Jean-Christophe Bailly. Le sujet énonciateur pratique tour à tour et tout à la fois des postures énonciatives diverses sinon contradictoires. Nous en indiquerons quelques-unes.

Discrètement autobiographe, comme nous avons pu le voir, le sujet auteur et narrateur se présente notamment dans ses fonctions de professeur de l'École Nationale Supérieur de la Nature et du Paysage de Blois. Fourmillant d'anecdotes relatives à cette fonction - comme c'est le cas à propos de la cité des Gibjoncs de Bourges - (463), le texte adopte un style professoral: «[...] la qualité principale du bariol, [...] est celle d'un élargissement du national, tant pour le pays récepteur que pour chaque habitant reçu, étant entendu que ce qui dès lors le reçoit, ce n'est pas tant une 'terre d'accueil' que, justement, une aire réservée, aux contours indécis, au sein de laquelle l'étrangeté (le fait d'être étranger) est la norme»(473). La voix qui parle, manifeste ici une attention pédagogique, sensible dans l'accumulation de détails (entre virgules ou entre parenthèses) visant à donner clarté à sa pensée, ou encore dans la prévention des erreurs possibles de son destinataire («ce n'est pas »), mais également une intention assertive (présent de vérité générale). Il s'agit de rendre sensible sinon palpable ce qui est pourtant comme une « hypothèse » conceptuelle.

Outre le professeur, ce sont également un amateur et un grand connaisseur d'art (se référant éclectiquement à Courbet, Rodin, Cranach, Cartier-Bresson, Matisse...), un homme de lettres habité par sa mémoire littéraire, un linguiste dans l'attention extrême qu'il porte au langage (qu'il s'agisse des noms propres - dont il déploie volontiers les «émanations »- ou de la justesse des termes et des tons choisis, ainsi lorsqu'il s'agit de parler de « République » ou de «peuple français »), un philosophe ou encore un naturaliste, qui s'expriment dans ce 
texte. Ne rencontrons-nous pas encore un collectionneur ${ }^{13}$ ? Le texte accumule, dans une libido quasi frénétique, les noms, les listes, les détails.

Dans la volonté encyclopédique dont le romantisme allemand a pu faire son principe $^{14}$, l'auteur se manifeste plus spécifiquement dans des compétences scientifiques plurielles et capables de se combiner entre elles avec la plus grande exigence de précision et de justesse : paysagiste (aussi attentif au parc de Lenôtre de Sceaux qu'aux jardins ouvriers de Saint-Etienne), géographe (lorsqu'il revisite les paysages façonnés par les guerres autour de Buzancy), architecte (livrant à son lecteur des schémas de la moulure française ondulée, considérant le bâtiment accueillant l'École des Beaux-Arts de Lorient, le Pont du Gard ou encore le Nemausus de Jean Nouvel à Nîmes), ou historien dans sa manière propre quoiqu'il s'en défende : «Chemin faisant, l'Histoire, avec ses grands et ses petits récits, ses simples bulles de sens et son grand vent, m'a rattrapé, prenant une importance que je n'avais pas prévue tout d'abord. Mais ce qui est venu ainsi à ma rencontre, ce n'est ni l'histoire des manuels ni celle des guides, c'est ce qu'il faudrait appeler une histoire des traces, dont le présent serait l'affleurement » (14).

Lancé à la recherche de «l'instantané mobile d'un pays ", l'auteur s'appuie alors sur les vestiges sensibles du passé pour tenter de mettre au jour une Histoire non pas canonique celle qui est enseignée dans les collèges et lycées -, mais une Histoire fondée sur une appréhension personnelle, susceptible pourtant de constituer un imaginaire collectif. Dès lors, c'est aussi un témoin qui vient comparaître. Déchiffrer le monde, lire les traces, sonder leur abîme, mener une enquête amenant à déplier les couches successives du temps, c'est autoriser sinon prôner le souvenir, mais sans nostalgie ni surtout «devoir de mémoire » institutionnalisé : «[...] être un souvenir n'est pas rien, c'est, ce pourrait être une activité, et même une activité continue »(395). Dans la hantise de l'oubli qui semble l'habiter, Bailly témoigne indirectement des événements funestes passés ${ }^{15}$ qui ont si fortement marqué le pays et, directement, la France contemporaine.

On reconnaîtra peut-être encore dans ce portrait multiple un moraliste (au sens de Pascal ou bien de Nietzsche) - notamment soucieux de convenance dans l'adéquation du ton à la matière traitée, par exemple lorsqu'il s'agit d'évoquer les champs de bataille de la première guerre mondiale ou lorsqu'il s'agit de légitimer le changement de fonction pour ce bâtiment qu'est la synagogue de Delme, où « l'histoire même du lieu empêche qu'on y fasse n'importe quoi » (186). La question de la convenance du ton à la matière traitée étant par 
ailleurs un des grands enjeux de la théorie romantique. Enfin, et avec des accents polémiques intenses, de fortes marques d'humeur, nous percevons surtout un homme engagé dans les circonstances du temps, qui se prononce de façon décidée et qui entend intervenir sur le cours des événements. L'œuvre de Bailly semble prendre par là la relève des engagements de parti-les distances prises avec ses engagements antérieurs n'ayant pas entraîné un reniement, mais un réajustement de la pensée et de la manière dans leur capacité d'intervention.

Où conduit le «fil rouge politique » dont l'auteur nous dit qu'il traverse tout son texte ? La critique politique, dans Le dépaysement comme aussi plus récemment dans le recueil de textes intitulé La phrase urbaine, porte essentiellement sur des questions d'urbanisme, d'occupation des sols, de tissage social, sur les rapports de l'homme à son milieu, dans une acception forte de l'écologie politique et, pour le dire plus généralement, sur les modalités d'une habitation poétique de la terre. Le motif holderlinien de l'habitation de la terre fait que pour Bailly les enjeux d'une poétique et d'une politique sont tout un. Des solutions pragmatiques précises sont alors proposées (ce plus nettement encore dans La phrase urbaine), pour remédier aux désastres constatés (par exemple, sur la question du zonage), et recomposer le paysage de l'habitation terrestre : «La perspective de ce grand chantier (qu'aucune des forces politiques agissant en France n'a l'air d'envisager sérieusement) ne consiste pas à projeter [...] une sorte de devenir-ville mimétique et nostalgique, ce dont il est (serait) question, c'est de logiques de pliages et redéploiements autonomes [...]. »(462).

Le questionnement initial sur la question de l'identité du pays « France » est laissé ouvert, une fois traité sous les multiples angles que nous avons essayé d'indiquer, et prémuni contre les récupérations qui menacent d'en fausser les termes, et l'ensemble du problème (récupérations gouvernementales au moment de la rédaction de l'ouvrage, nationalistes, communautaristes, extrémistes): «Mais ce peuple français que désigne de façon neutre, générique et hautaine l'inscription d'inspiration robespierriste, où est-il, que fait-il ? Existe-il encore vraiment quelque chose qui, dans le paysage éclaté de la France d'aujourd'hui lui ressemble ? Et doit-il y avoir une ressemblance ? (Bailly, Dépaysement, 396).

Pour atteindre «l'image d'une totalité énigmatique éclatée dans chaque méandre de l'existence [...]»(Légende, 17), Jean-Christophe Bailly présente en somme un ensemble d'attitudes à adopter. Un double postulat le soutient : d'une part, la possibilité de totaliser 
mais de façon seulement virtuelle un ensemble disparate et, d'autre part, celle de dégager les correspondances secrètes tissant cette totalité. Le microcosme que constitue son œuvre, est ainsi en parfaite résonance avec le macrocosme que forme son objet. En mettant au jour les ligatures d'un tissu que le tissu pourra se laisser palper, l'ambition politique et sociale de Bailly se double d'une ambition littéraire qui en est l'exact reflet. Définir une totalité indéfinissable et mobile - l'identité française -, nécessite en effet de faire intervenir l'esprit $\mathrm{du}$ « roman colossal », afin de ne jamais clore ni fixer son objet. Euvre inclassable répondant à l'impératif de ne pas se laisser saisir et d'être en instance de départ permanent, dans le renouvellement constant de l'inachevé, ces « voyages en France » élaborent in fine leur unité dans l'incitation faite au lecteur. Invitation au dépaysement littéraire, l'œuvre de Bailly est aussi et surtout, parce que le geste créatif durant le cheminement de l'enquête se veut fondateur, appel à un re-paysement du paysage textuel que son lecteur croyait pourtant fixé.

\section{Ouvrages cités}

Bailly, Jean-Christophe. Le dépaysement. Voyages en France. Paris : Seuil, « Points », 2011.

---. La phrase urbaine. Paris : Seuil, « Fiction \& Cie », 2013.

---. La légende dispersée. Anthologie. Paris : Christian Bourgeois, « Détroits », 2001 [1976].

Montaigne, Michel (de). Les essais. Paris : Gallimard, « Pléiade», 2007 [1595].

Novalis. Le brouillon général. Tr. Olivier Schefer. Paris : Allia, 2000.

${ }^{1}$ L'image de la ville assimilée à un système de signes, un langage, une grammaire, est abondamment développée dans La Phrase urbaine.

${ }^{2}$ Le passage de Novalis qui nous intéresse figure dans l'anthologie La Légende dispersée (120) : «Rien n’est plus romantique que ce qu'on nomme communément monde et destin. - Nous vivons dans un roman colossal (en grand et en petit). Considération de ce qui se passe autour de nous. Orientation romantique. Appréciation et étude de la vie humaine » (Novalis, 1393, IV - 562).

3 Notons que Novalis «désubstantialise » la matière lourde et fixe, pour en faire émerger, en « exciter»les forces primordiales. À ce propos, voir Novalis, Brouillon.

${ }^{4}$ L'écriture de Bailly définie comme un agrégat de départs n'est pas sans évoquer le fait que « [c]e qui frappe quand on lit les biographies de la plupart des romantiques allemands, c'est leur refus de se fixer. »Voir Légende, 29.

5 Voir Dépaysement, 338 : la «dormance» serait «une capacité d'éveil ou de réveil qui se maintient en traversant le temps. » Le terme est rapproché de la «partance» dans La Phrase urbaine, 151: «Et cette «partance » (comme on dit dormance pour les graines) n'est rien d'autre que ce qui tient l'être dans l'ouvert, ce qui le tire hors de la somme et de la sommation ».

${ }^{6}$ Rappelons que pour Bailly, le romantisme allemand «est la pensée de l'inachèvement même », en cela liée à la pratique de la forme brève. Voir Légende, 24.

${ }^{7}$ L'amitié et la «communauté de pensée » sans allégeance sont au fondement du romantisme allemand tel que l'analyse notre auteur. Voir Légende, 30.

${ }^{8}$ Le dépaysement, voir le chapitre nommé « L'hypothèse du bariol », 468 et seq.

9 «Utopia povera » dans La phrase urbaine, p. 140 notamment.

${ }^{10}$ L'auteur souligne.
} 
${ }^{11}$ L'expression s'inspire de « La grammaire générative des jambes » (Phrase urbaine, 19-40).

${ }^{12}$ C'est par exemple le cas avec ce type d'insertion : «(elle a déjà été croisée ici dans le chapitre « Le voyage de la fève »)»(Dépaysement, 276).

${ }^{13}$ Notons que dans la pensée de Walter Benjamin, à laquelle Bailly est particulièrement attaché, collection (rapportée au monde de l'enfance) et flânerie sont liées.

14 «La philosophie, l'art, la religion, la politique, le mythe, la fiction, la médecine, le romantisme allemand allait les aborder avec passion en s'y jetant, mais ce mouvement n'était pas un abandon: les limites de chaque domaine, les limites du langage propres à chaque domaine s'estompaient devant la force d'une exigence plus grande encore. »(Bailly, Légende, 18)

L'auteur est extrêmement perméable aux traces les plus funestes et angoissantes, celles des guerres notamment, récentes ou datant du «pays des Éduens» : un «chant de l'archive » permet en effet de «faire partir les phrases d'objets ruraux que nous connaissons vers de très lointaines aires de résonance, d'où quelque chose d'inquiet ou, plus précisément, quelque chose qui est lié à la peur, ne se défait jamais complètement » (Dépaysement, 409). 